\title{
Vector shock soliton and the Hirota bilinear method
}

\author{
Oktay Pashaev *, Gamze Tanoğlu \\ Department of Mathematics, Izmir Institute of Technology, Gulbahce koyu, Urla, Izmir 35430, Turkey
}

Accepted 9 December 2004

\begin{abstract}
The Hirota bilinear method is applied to find an exact shock soliton solution of the system reaction-diffusion equations for $n$-component vector order parameter, with the reaction part in form of the third order polynomial, determined by three distinct constant vectors. The bilinear representation is derived by extracting one of the vector roots (unstable in general), which allows us reduce the cubic nonlinearity to a quadratic one. The vector shock soliton solution, implementing transition between other two roots, as a fixed points of the potential from continuum set of the values, is constructed in a simple way. In our approach, the velocity of soliton is fixed by truncating the Hirota perturbation expansion and it is found in terms of all three roots. Shock solitons for extensions of the model, by including the second order time derivative term and the nonlinear transport term are derived. Numerical solutions illustrating generation of solitary wave from initial step function, depending of the polynomial roots are given.
\end{abstract}

(c) 2005 Elsevier Ltd. All rights reserved.

\section{Introduction}

Solutions of nonlinear evolution equations appearing as travelling waves play an distinctive role in nonlinear phenomena [1,2]. These solutions are connected with genetic waves in biology, vibrations in physics, phase transitions in material science, so on. Travelling wavefront solutions effect a smooth transition between two, space independent (homogeneous) steady states of the system. The speed of such waves is an important characteristic for applications, related with values of these steady states. In mathematics, a number of techniques has been developed to obtain the travelling wave solution for nonlinear evolution equations [3-5]. One of the most popular classical methods is the Lie method and its generalizations, as nonclassical method of group-invariant solutions, direct method for finding symmetry reductions, etc. [3], based on using the Lie symmetry of a given PDE in order to construct exact solution. On the other hand, it is known that some non-integrable nonlinear PDEs (for example, the well known Fisher equation) have poor Lie symmetry, being invariant only under the time and space translations. The Lie method is not efficient for such PDEs and become cumbersome [4]. The other method, based on travelling wave ansatz [5], reduces nonlinear PDE to a nonlinear ODE. But in ODE system, the travelling wave speed is an unknown parameter that must be fixed by the analysis and choosing a special trial trajectory [2]. It appears that if resulting ODE is of the Painleve type, then it can be solved explicitly, leading to exact solution of the original equation. This way Ablowitz and Zeppetella [6] obtained

\footnotetext{
* Corresponding author.

E-mail addresses: oktaypashaev@iyte.edu.tr (O. Pashaev), gamzetanoglu@iyte.edu.tr (G. Tanoğlu).
} 
an exact travelling wave solution of Fisher's equation by finding the special wave speed for which the resulting ODE is of the Painleve type. However, the method seems does not allow extensions to more than one travelling waves, higher space dimensions and multi-component order parameter. In the case of a multi-species (phases, cells, reactants, populations, and so on) system, where reaction term has several steady states, one should expect similar travelling wave solution that join steady states. The variety of spatial wave phenomena in multi-species reaction-diffusion system and in space dimensions more than one is very much richer than in single species models [1]. But since the dimension of phase space for analysis of travelling wavefront solutions increases, such multi-species solutions are more difficult to determine analytically.

From another site, during the last 30 years the direct method proposed by Hirota becomes a powerful tool for constructing multisoliton solutions to integrable nonlinear evolution equations [7]. This, relatively simple and algebraic rather than analytic method, allows one to avoid many analytic difficulties of more sophisticated the inverse scattering method. Moreover, it is deeply related with Plücker coordinates of Grassmanians, quantum theory of fermions, $\tau$ functions and vertex operator representation of infinite-dimensional algebras [8]. The general idea of the method is first to transform the nonlinear equation under consideration into a bilinear equation or system of equations, and then use the formal power series expansion to solve it. For integrable systems the series admit exact truncation for an arbitrary number of solitons. While for periodic solutions it includes an infinite number of terms. We will see below that the truncation of Hirota's perturbation series for non-integrable case, similarly to the Painleve reduction [6], fixes the velocity of soliton.

In the present paper we demonstrate effectiveness of the Hirota method for constructing shock soliton solution of $n$ component reaction-diffusion equation in three space dimensions. We consider equation for the vector order parameter $\mathbf{U}(x, y, z)=\left(U^{1}(x, y, z), U^{2}(x, y, z), \ldots, U^{n}(x, y, z)\right)$ and cubic nonlinear reaction term

$$
\frac{\partial \mathbf{U}}{\partial t}=\nabla^{2} \mathbf{U}-\left(\mathbf{U}-\mathbf{a}_{1}, \mathbf{U}-\mathbf{a}_{2}\right)\left(\mathbf{U}-\mathbf{a}_{3}\right),
$$

where $\mathbf{a}_{j}=\left(a_{j}^{1}, a_{j}^{2}, \ldots, a_{j}^{n}\right) \in R^{n},(j=1,2,3)$, are three distinct constant vectors, and $\left(\mathbf{U}-\mathbf{a}_{1}, \mathbf{U}-\mathbf{a}_{2}\right) \equiv \sum_{i=1}^{n}\left(U^{i}-a_{1}^{i}\right)$ $\left(U^{i}-a_{2}^{i}\right)$ means the Euclidean scalar product of vectors $\mathbf{U}-\mathbf{a}_{1}$ and $\mathbf{U}-\mathbf{a}_{2}$ and $\nabla^{2}=\Delta=\partial^{2} / \partial x^{2}+\partial^{2} / \partial y^{2}+\partial^{2} / \partial z^{2}$ is the Laplace operator. In the scalar case, when $n=1$, and in the one space dimension, for different choices of parameters $a_{1}, a_{2}, a_{3}$ the model reduces to the well known nonlinear diffusion equations appearing in a different fields sometimes with different names: the Fitzhugh-Nagumo equation $\left(a_{1}=0, a_{2}=1, a_{3}=a\right)$ arising in population genetics [9] and models the transmission of nerve impulse [10,11], autocatalytic chemical reaction model introduced by Schlögl [12], generalized Fisher equation [2], Newell-Whitehead equation [14] or Kolmogorov-Petrovsky-Piscounov equation [15] $\left(a_{1}=0, a_{2}=1, a_{3}=-1\right)$, Huxley equation $\left(a_{1}=0, a_{2}=0, a_{3}=1\right)$.

The method allows us to consider extensions of the above model to nonlinear wave equation with dissipation in three dimensions

$$
\frac{\partial^{2} U}{\partial t^{2}}+\gamma \frac{\partial U}{\partial t}=\nabla^{2} U-\left(U-a_{1}\right)\left(U-a_{2}\right)\left(U-a_{3}\right)
$$

and to the nonlinear wave equation with nonlinear transport term

$$
\alpha U \frac{\partial U}{\partial x}+\beta \frac{\partial^{2} U}{\partial t^{2}}+\gamma \frac{\partial U}{\partial t}=\frac{\partial^{2} U}{\partial x^{2}}-\left(U-a_{1}\right)\left(U-a_{2}\right)\left(U-a_{3}\right),
$$

where $a_{1}, a_{2}, a_{3}$ are distinct real numbers and $\alpha, \beta, \gamma$ are constants. For simplicity, in the last two equations the dimension for order parameter space is chosen to be one, $n=1$. To bilinearize these equations with cubic nonlinearity, we propose in the Hirota ansatz to extract one of the roots as a background field. Then, a solution of bilinear equations gives the travelling wave, implementing transition between other two roots and moving with velocity, depending on values of all three roots. We note that more restrictive ansatz in terms of only one function $U=f_{x} / f$ [13], like for the $\mathrm{KdV}$ equation, does not allow one to find bilinear equations in terms of the Hirota derivatives [16,17] and extend the approach to the multidimensional case or for the vector order parameter.

The paper is organized as follows. In Section 2, by the Hirota ansatz Eq. (1) is transformed into the bilinear system of $n+1$ differential equations, which we solve exactly using Hirota's perturbation approach. In Section 3, by adding the second derivative term with respect to the time, we study multidimensional nonlinear wave equation with dissipation (2). We show that if the background root of our ansatz is the mean value of the other two roots, the velocity parameter is vanishing and solitary wave is static. In Section 4, extension of the model by the nonlinear transport term is considered. We show that even in the mean value case our solution now is not static. Then we present numerical solutions generating shock soliton from initial step function. In conclusion, we summarize our results and discuss possible extensions to other equations. 


\section{Vector reaction-diffusion equation}

To reduce Eq. (1) with cubic nonlinearity and three distinct roots $\mathbf{a}_{1}, \mathbf{a}_{2}, \mathbf{a}_{3}$, to the bilinear form, we have to modify the standard Hirota ansatz by extracting one of the vector roots. The solution of the problem is assumed to have a form

$$
\mathbf{U}=\mathbf{a}_{3}+\frac{\mathbf{g}}{f}
$$

where $\mathbf{g}(\mathbf{x}, t)$ is a $n$-component real vector function and $f(\mathbf{x}, t)$ is a real function. In the bilinear approach all derivatives with respect to the dependent variables in Eq. (1) are expressed as

$$
\begin{aligned}
& \mathbf{U}_{t}=\frac{D_{t}(\mathbf{g} \cdot f)}{f^{2}} \\
& \nabla^{2} \mathbf{U}=\frac{D_{x}^{2}(\mathbf{g} \cdot f)}{f^{2}}+\frac{D_{y}^{2}(\mathbf{g} \cdot f)}{f^{2}}+\frac{D_{z}^{2}(\mathbf{g} \cdot f)}{f^{2}}-\frac{\mathbf{g}}{f} \frac{D_{x}^{2}(f \cdot f)}{f^{2}}-\frac{\mathbf{g}}{f} \frac{D_{y}^{2}(f \cdot f)}{f^{2}}-\frac{\mathbf{g}}{f} \frac{D_{z}^{2}(f \cdot f)}{f^{2}},
\end{aligned}
$$

where the Hirota derivative according to $x_{i}$ is defined as

$$
D_{x_{i}}^{n}(a \cdot b)=\left.\left(\frac{\partial}{\partial x_{i}}-\frac{\partial}{\partial x_{i}^{\prime}}\right)^{n}\left(a(\mathbf{x}) b\left(\mathbf{x}^{\prime}\right)\right)\right|_{\mathbf{x}=\mathbf{x}^{\prime}} .
$$

After substituting Eqs. (5) into Eq. (1), the following expression is obtained:

$$
\begin{aligned}
& \frac{D_{t}(\mathbf{g} \cdot f)}{f^{2}}-\frac{D_{x}^{2}(\mathbf{g} \cdot f)}{f^{2}}-\frac{D_{y}^{2}(\mathbf{g} \cdot f)}{f^{2}}-\frac{D_{z}^{2}(\mathbf{g} \cdot f)}{f^{2}}+\frac{\mathbf{g}}{f} \frac{D_{x}^{2}(f \cdot f)}{f^{2}}+\frac{\mathbf{g}}{f} \frac{D_{y}^{2}(f \cdot f)}{f^{2}}+\frac{\mathbf{g} \frac{D_{z}^{2}(f \cdot f)}{f}}{f^{2}} \\
& +\frac{\mathbf{g}}{f}\left(\left(\frac{\mathbf{g}}{f}-\left(\mathbf{a}_{1}-\mathbf{a}_{3}\right)\right),\left(\frac{\mathbf{g}}{f}-\left(\mathbf{a}_{2}-\mathbf{a}_{3}\right)\right)\right)=0 .
\end{aligned}
$$

Since by Eq. (4) instead of one $n$-dimensional vector function $\mathbf{U}$ we introduced $n$-dimensional vector function $\mathbf{g}$ and one more scalar function $f$, we have freedom to decouple this system of $n$-equations as the bilinear system of $n+1$ equations

$$
\begin{aligned}
& \left(D_{t}-D_{x}^{2}-D_{y}^{2}-D_{z}^{2}\right)(\mathbf{g} \cdot f)=0, \\
& \left(D_{x}^{2}+D_{y}^{2}+D_{z}^{2}+\left(\tilde{\mathbf{a}}_{1}, \tilde{\mathbf{a}}_{2}\right)\right)(f \cdot f)=-(\mathbf{g}, \mathbf{g})+\left(\left(\tilde{\mathbf{a}}_{1}+\tilde{\mathbf{a}}_{2}\right), \mathbf{g}\right) f,
\end{aligned}
$$

where $\tilde{\mathbf{a}}_{1} \equiv \mathbf{a}_{1}-\mathbf{a}_{3}$ and $\tilde{\mathbf{a}}_{2} \equiv \mathbf{a}_{2}-\mathbf{a}_{3}$. To solve this system in the Hirota method, the functions $f$ and vector function $\mathbf{g}$ suppose to have form of the formal perturbation series in a parameter $\epsilon$

$$
\begin{aligned}
& f=1+\epsilon f_{1}+\epsilon^{2} f_{2}+\cdots, \\
& \mathbf{g}=\mathbf{g}_{0}+\epsilon \mathbf{g}_{1}+\epsilon^{2} \mathbf{g}_{2}+\cdots
\end{aligned}
$$

Substituting (9) into the system (8) and equating coefficients of the same powers of $\epsilon$ converts (8) into a sequence of the zeroth, first, second and higher order, bilinear equations

$$
\begin{aligned}
& \left(D_{t}-D_{x}^{2}-D_{y}^{2}-D_{z}^{2}\right)\left(\mathbf{g}_{0} \cdot 1\right)=0 \\
& \left(D_{x}^{2}+D_{y}^{2}+D_{z}^{2}+\left(\tilde{\mathbf{a}}_{1}, \tilde{\mathbf{a}}_{2}\right)\right)(1 \cdot 1)=-\left(\mathbf{g}_{0}, \mathbf{g}_{0}\right)+\left(\tilde{\mathbf{a}}_{1}+\tilde{\mathbf{a}}_{2}, \mathbf{g}_{0}\right) \\
& \left(D_{t}-D_{x}^{2}-D_{y}^{2}-D_{z}^{2}\right)\left(\mathbf{g}_{0} \cdot f_{1}+\mathbf{g}_{1} \cdot 1\right)=0 \\
& \left(D_{x}^{2}+D_{y}^{2}+D_{z}^{2}+\left(\tilde{\mathbf{a}}_{1}, \tilde{\mathbf{a}}_{2}\right)\right)\left(2 \cdot f_{1}\right)=-2\left(\mathbf{g}_{0}, \mathbf{g}_{1}\right)+\left(\tilde{\mathbf{a}}_{1}+\tilde{\mathbf{a}}_{2}, \mathbf{g}_{0} f_{1}+\mathbf{g}_{1}\right) \\
& \left(D_{t}-D_{x}^{2}-D_{y}^{2}-D_{z}^{2}\right)\left(\mathbf{g}_{0} \cdot f_{2}+\mathbf{g}_{1} \cdot f_{1}+\mathbf{g}_{2} \cdot 1\right)=0 \\
& \left(D_{x}^{2}+D_{y}^{2}+D_{z}^{2}+\left(\tilde{\mathbf{a}}_{1}, \tilde{\mathbf{a}}_{2}\right)\right)\left(2 \cdot f_{2}+f_{1} \cdot f_{1}\right)=-2\left(\mathbf{g}_{0}, \mathbf{g}_{2}\right)-\left(\mathbf{g}_{1}, \mathbf{g}_{1}\right) \\
& +\left(\tilde{\mathbf{a}}_{1}+\tilde{\mathbf{a}}_{2}, \mathbf{g}_{0} f_{2}+\mathbf{g}_{1} f_{1}+\mathbf{g}_{2}\right) \\
& \ldots
\end{aligned}
$$


We assume that all components of vector $\mathbf{g}_{0}$ are constants, then the first equation (10a) is satisfied automatically. From the second equation we get

$$
\left(\mathbf{g}_{0}, \mathbf{g}_{0}\right)-\left(\tilde{\mathbf{a}}_{1}+\tilde{\mathbf{a}}_{2}, \mathbf{g}_{0}\right)+\left(\tilde{\mathbf{a}}_{1}, \tilde{\mathbf{a}}_{2}\right)=\left(\mathbf{g}_{0}-\tilde{\mathbf{a}}_{1}, \mathbf{g}_{0}-\tilde{\mathbf{a}}_{2}\right)=0 .
$$

Solutions of the last equation can be found as: (1) $\mathbf{g}_{0}=\tilde{\mathbf{a}}_{1}$, (2) $\mathbf{g}_{0}=\tilde{\mathbf{a}}_{2}$ and (3) $\left(\mathbf{g}_{0}-\tilde{\mathbf{a}}_{1}\right) \perp\left(\mathbf{g}_{0}-\tilde{\mathbf{a}}_{2}\right)$. For simplicity we assume that $\mathbf{g}_{0}=\tilde{\mathbf{a}}_{1}$. As a next step we are going to find the first order solutions, $\mathbf{g}_{1}$ and $f_{1}$. Then Eqs. (11) reduce to a linear system

$$
\begin{aligned}
& \tilde{\mathbf{a}}_{1}\left(-\partial_{t}-\Delta\right) f_{1}+\left(\partial_{t}-\Delta\right) \mathbf{g}_{1}=0, \\
& 2 \Delta f_{1}+2\left(\tilde{\mathbf{a}}_{1}, \tilde{\mathbf{a}}_{2}\right) f_{1}+2\left(\tilde{\mathbf{a}}_{1}, \mathbf{g}_{1}\right)-\left(\tilde{\mathbf{a}}_{1}+\tilde{\mathbf{a}}_{2}, \tilde{\mathbf{a}}_{1} f_{1}+\mathbf{g}_{1}\right)=0,
\end{aligned}
$$

where $\Delta$ denotes the three dimensional Laplace operator. Nontrivial solution of this system suppose to have the form

$$
\mathbf{g}_{1}=\mathbf{a} e^{\eta_{1}}, \quad f_{1}=b e^{\eta_{1}}
$$

where $\mathbf{a}=\left(a^{1}, a^{2}, \ldots, a^{n}\right)$ and $b$ are $n+1$ constants, $\eta_{1}=\mathbf{k x}+\omega t+\delta_{0}$. Here $\mathbf{k x}=k_{x} x+k_{y} y+k_{z} z$ means the three dimensional scalar product. Unknown constants, the wave vector $\mathbf{k}$ and frequency $\omega$ are fixed by a dispersion relation, while $n$ constants $\left(a^{1}, a^{2}, \ldots, a^{n}\right)$ are fixed by Eqs. (14a). After substituting (15) into the system (14) for variables $\left(a^{1}, a^{2}, \ldots, a^{n}\right)$ and $b$ we have $(n+1) \times(n+1)$ homogeneous linear algebraic system:

$$
A \xi=\left[\begin{array}{ccccc}
\left(\omega-\mathbf{k}^{2}\right) & 0 & 0 & . & \tilde{a}_{1}{ }^{1}\left(-\omega-\mathbf{k}^{2}\right) \\
0 & \left(\omega-\mathbf{k}^{2}\right) & 0 & . & \tilde{a}_{1}{ }^{2}\left(-\omega-\mathbf{k}^{2}\right) \\
\cdot & \cdot & \cdot & \cdot & \cdot \\
0 & 0 & \cdot & \left(\omega-\mathbf{k}^{2}\right) & \tilde{a}_{1}{ }^{n}\left(-\omega-\mathbf{k}^{2}\right) \\
\left(\tilde{a}_{1}{ }^{1}-{\tilde{a_{2}}}^{1}\right) & \cdot & . & \left({\tilde{a_{1}}}^{n}-{\tilde{a_{2}}}^{n}\right) & 2 \mathbf{k}^{2}+\left(\tilde{\mathbf{a}}_{1}, \tilde{\mathbf{a}}_{2}-\tilde{\mathbf{a}}_{1}\right)
\end{array}\right]\left[\begin{array}{c}
a^{1} \\
a^{2} \\
\vdots \\
a^{n} \\
b
\end{array}\right]=\left[\begin{array}{c}
0 \\
0 \\
\vdots \\
0 \\
0
\end{array}\right] .
$$

Nontrivial solution of this system of equations exists only if $\operatorname{Det}(A)=0$. This determinant can be evaluated by expansion along the last row, so that we have expression

$$
\operatorname{Det}(A)=\left(\omega-\mathbf{k}^{2}\right)^{n-1}\left[\left(\omega-\mathbf{k}^{2}\right)\left(2 \mathbf{k}^{2}+\left(\tilde{\mathbf{a}}_{1}, \tilde{\mathbf{a}}_{2}-\tilde{\mathbf{a}}_{1}\right)\right)-\left(-\omega-\mathbf{k}^{2}\right) \sum_{i=1}^{n} \tilde{\mathbf{a}}_{1}^{i}\left(\tilde{\mathbf{a}}_{1}-\tilde{\mathbf{a}}_{2}\right)^{i}\right] .
$$

It gives us the following dispersion relations:

$$
\omega=\mathbf{k}^{2}
$$

and

$$
\omega=\mathbf{k}^{2}+\left(\tilde{\mathbf{a}}_{1}, \tilde{\mathbf{a}}_{2}-\tilde{\mathbf{a}}_{1}\right) .
$$

The dispersion relation (17) gives us a constant solution, thus we use dispersion (18) for the further calculations. Solving the first $n$ equations in the above $(n+1) \times(n+1)$ linear algebraic system we have:

$$
\mathbf{a}=b \gamma_{1} \tilde{\mathbf{a}}_{1}
$$

where

$$
\gamma_{1}=\frac{2 \mathbf{k}^{2}+\left(\tilde{\mathbf{a}}_{1}, \tilde{\mathbf{a}}_{2}-\tilde{\mathbf{a}}_{1}\right)}{\left(\tilde{\mathbf{a}}_{1}, \tilde{\mathbf{a}}_{2}-\tilde{\mathbf{a}}_{1}\right)},
$$

so that

$$
\mathbf{g}_{1}=b \gamma_{1} \tilde{\mathbf{a}}_{1} e^{\eta_{1}}, \quad f_{1}=b e^{\eta_{1}} .
$$

Substituting $\mathbf{g}_{1}, f_{1}$ to the first system of Eqs. (12a) and using property of bilinear Hirota operators [7]

$$
\left(D_{t}-D_{x}^{2}\right)\left(\mathbf{g}_{1} \cdot f_{1}\right)=b^{2} \gamma_{1} \tilde{\mathbf{a}}_{1}\left(D_{t}-D_{x}^{2}\right)\left(e^{\eta_{1}} \cdot e^{\eta_{1}}\right)=0
$$

for $\mathbf{g}_{2}, f_{2}$ we find the following set of equations

$$
\tilde{\mathbf{a}}_{1}\left(-\partial_{t}-\Delta\right) f_{2}+\left(\partial_{t}-\Delta\right) \mathbf{g}_{2}=0,
$$


similar to the first system (14a). Simplest solution for these equations is the trivial one $\mathbf{g}_{2}=\mathbf{0}$ and $f_{2}=0$. Then, from the last Eq. (12a) we find additional constraint on $\mathbf{g}_{1}, f_{1}$ :

$$
\left(\tilde{\mathbf{a}}_{1}, \tilde{\mathbf{a}}_{2}\right) f_{1}^{2}=-\left(\mathbf{g}_{1}, \mathbf{g}_{1}\right)+\left(\tilde{\mathbf{a}}_{1}+\tilde{\mathbf{a}}_{2}, \mathbf{g}_{1}\right) f_{1} .
$$

Substituting solution (21) into Eq. (24) results in the following relation:

$$
\left(\tilde{\mathbf{a}}_{1}, \tilde{\mathbf{a}}_{2}\right)=-\left(\tilde{\mathbf{a}}_{1}, \tilde{\mathbf{a}}_{1}\right) \gamma_{1}^{2}+\left(\tilde{\mathbf{a}}_{1}+\tilde{\mathbf{a}}_{2}, \tilde{\mathbf{a}}_{1}\right) \gamma_{1},
$$

which can be written in the form:

$$
\left(\gamma_{1}-1\right)\left(\tilde{\mathbf{a}}_{1},\left(\gamma_{1} \tilde{\mathbf{a}}_{1}-\tilde{\mathbf{a}}_{2}\right)\right)=0 .
$$

Assuming $\gamma_{1}=1$, leads to the trivial result $\mathbf{k}=0$. Thus, if $\gamma_{1} \neq 1$ from Eq. (26) the following nontrivial solution can be found:

$$
\gamma_{1}=\frac{\left(\tilde{\mathbf{a}}_{1}, \tilde{\mathbf{a}}_{2}\right)}{\left(\tilde{\mathbf{a}}_{1}, \tilde{\mathbf{a}}_{1}\right)} .
$$

Combining this equation with Eq. (20), we find restrictions on allowed values of the length for the wave vector $\mathbf{k}$

$$
\mathbf{k}^{2}=\frac{\left(\tilde{\mathbf{a}}_{1}, \tilde{\mathbf{a}}_{2}-\tilde{\mathbf{a}}_{1}\right)^{2}}{2\left(\tilde{\mathbf{a}}_{1}, \tilde{\mathbf{a}}_{1}\right)},
$$

or

$$
|\mathbf{k}|=\frac{\left|\left(\tilde{\mathbf{a}}_{1}, \tilde{\mathbf{a}}_{2}-\tilde{\mathbf{a}}_{1}\right)\right|}{\sqrt{2}\left|\tilde{\mathbf{a}}_{1}\right|} .
$$

This equation shows that comparing with the one dimensional case the wave vector now belongs to the three dimensional sphere with fixed radius. Next, after substituting Eq. (28) into Eq. (18), the following explicit form for the frequency is obtained

$$
\omega=\frac{\left(\tilde{\mathbf{a}}_{1}, \tilde{\mathbf{a}}_{2}-\tilde{\mathbf{a}}_{1}\right)\left(\tilde{\mathbf{a}}_{1}, \tilde{\mathbf{a}}_{2}+\tilde{\mathbf{a}}_{1}\right)}{2\left(\tilde{\mathbf{a}}_{1}, \tilde{\mathbf{a}}_{1}\right)} .
$$

Finally, the velocity vector is given by formula:

$$
\mathbf{v}=-\omega \frac{\mathbf{k}}{|\mathbf{k}|^{2}} \text {. }
$$

With the wave vector $\mathbf{k}$ given by Eq. (29) and frequency $\omega$ given by Eq. (30) for the speed of solitary wave we have the expression in terms of three vectors $\mathbf{a}_{1}, \mathbf{a}_{2}, \mathbf{a}_{3}$ as

$$
|\mathbf{v}|=\frac{\left(\mathbf{a}_{1}-\mathbf{a}_{3}, \mathbf{a}_{1}+\mathbf{a}_{2}-2 \mathbf{a}_{3}\right)}{\sqrt{2}\left|\mathbf{a}_{1}-\mathbf{a}_{3}\right|} .
$$

It is easy to show that each bilinear equation, which has order greater than 2, has simple solution as, $\mathbf{g}_{i}=\mathbf{0}$ and $f_{i}=0$, for $i>2$. Therefore, we have only finite number of terms in the expansion (9). After substituting $f$ and $\mathbf{g}$ in Eq. (4), we find the following exact solution of our problem

$$
\mathbf{U}=\mathbf{a}_{3}+\left(\mathbf{a}_{1}-\mathbf{a}_{3}\right) \frac{1+\gamma_{1} e^{\eta_{1}}}{1+e^{\eta_{1}}},
$$

where $\gamma_{1}=\left(\tilde{\mathbf{a}}_{1}, \tilde{\mathbf{a}}_{2}\right) /\left(\tilde{\mathbf{a}}_{1}, \tilde{\mathbf{a}}_{1}\right)$. We note that the constant $b$ appearing only in front of exponential terms can be absorbed by the arbitrary constant $\delta_{0}$ in Eq. (15) and leads just to shift of the soliton's origin. In terms of original vectors $\mathbf{a}_{1}, \mathbf{a}_{2}, \mathbf{a}_{3}$ solution acquires the final form

$$
\mathbf{U}=\mathbf{a}_{3}+\frac{\mathbf{a}_{1}-\mathbf{a}_{3}}{\left|\mathbf{a}_{1}-\mathbf{a}_{3}\right|^{2}} \frac{\left(\left(\mathbf{a}_{1}-\mathbf{a}_{3}\right),\left(\mathbf{a}_{1}-\mathbf{a}_{3}+\left(\mathbf{a}_{2}-\mathbf{a}_{3}\right) e^{\eta_{1}}\right)\right)}{1+e^{\eta_{1}}} .
$$

This solution is the shock-solitary wave with asymptotics $\mathbf{U} \rightarrow \mathbf{a}_{1}$, for $\eta_{1} \rightarrow-\infty$ and $\mathbf{U} \rightarrow\left(1-\gamma_{1}\right) \mathbf{a}_{3}+\gamma_{1} \mathbf{a}_{1}$, for $\eta_{1} \rightarrow+\infty$, at a fixed time. As easy to see, these asymptotic solutions are the stationary $\left(\mathbf{U}_{t}=0\right)$ homogeneous $\left(\mathbf{U}_{x}=\right.$ $\mathbf{U}_{y}=\mathbf{U}_{z}=0$ ) solutions of Eq. (1). The parameter $\gamma_{1}$ in this solution has meaning of the ratio $\gamma_{1}=\left(\left|\tilde{\mathbf{a}}_{2}\right| /\left|\tilde{\mathbf{a}}_{1}\right|\right) \cos \alpha$, where 
$0 \leqslant \alpha \leqslant \pi$ is the angle between vectors $\tilde{\mathbf{a}}_{1}, \tilde{\mathbf{a}}_{2}$. Since Eq. (26) has infinite number of solutions, our shock soliton interpolates between the "vacuum" solution determined by vector $\mathbf{a}_{1}$ and the solution with vector $\mathbf{a}_{3}+\left(\tilde{\mathbf{a}}_{1}, \tilde{\mathbf{a}}_{2}\right) \tilde{\mathbf{a}}_{1} /\left|\tilde{\mathbf{a}}_{1}\right|^{2}$ which is valued in the continuum set.

If in Eq. (13) for $\mathbf{g}_{0}$ we choose another root $\mathbf{g}_{0}=\tilde{\mathbf{a}}_{2}$, then we have another shock soliton solution of Eq. (1)

$$
\mathbf{U}=\mathbf{a}_{3}+\left(\mathbf{a}_{2}-\mathbf{a}_{3}\right) \frac{1+\gamma_{2} e^{\eta_{1}}}{1+e^{\eta_{1}}},
$$

where $\gamma_{2}=\left(\tilde{\mathbf{a}}_{1}, \tilde{\mathbf{a}}_{2}\right) /\left(\tilde{\mathbf{a}}_{2}, \tilde{\mathbf{a}}_{2}\right)$. Solution (35) has asymptotics $\mathbf{U} \rightarrow \mathbf{a}_{2}$, for $\eta_{1} \rightarrow-\infty$ and $\mathbf{U} \rightarrow\left(1-\gamma_{2}\right) \mathbf{a}_{3}+\gamma_{2} \mathbf{a}_{2}$, for $\eta_{1} \rightarrow+\infty$, at a fixed time. The parameter $\gamma_{2}$ has meaning of the ratio $\gamma_{2}=\left(\left|\tilde{\mathbf{a}}_{1}\right| /\left|\tilde{\mathbf{a}}_{2}\right|\right) \cos \alpha$, where $\alpha$ as above for the $\gamma_{1}$, is the angle between vectors $\tilde{\mathbf{a}}_{1}, \tilde{\mathbf{a}}_{2}$.

In the scalar case, when $n=1$, the wave number (29), frequency (30) and velocity (31) (as well as the form of our solution (33)) reduces to the known ones

$$
k=\mp \frac{a_{2}-a_{1}}{\sqrt{2}}, \quad \omega=\frac{\left(a_{2}-a_{3}\right)^{2}-\left(a_{1}-a_{3}\right)^{2}}{2}, \quad v= \pm \frac{a_{1}+a_{2}-2 a_{3}}{\sqrt{2}} .
$$

\section{Dissipative nonlinear wave equation}

In this section, we consider the nonlinear wave equation with dissipation (2), which can be considered as an extension of the reaction-diffusion equation (1) by adding the second order time derivative term. For simplicity we restrict here the analysis by the scalar equation with $n=1$. To apply the Hirota bilinearization we use the ansatz

$$
U=a_{3}+\frac{g}{f}
$$

the same as in the scalar case of Eq. (4). In terms of Hirota derivatives (5) we have the following bilinear system of equations

$$
\begin{aligned}
& \left(D_{t}^{2}+\gamma D_{t}-D_{x}^{2}-D_{y}^{2}-D_{z}^{2}\right)(g \cdot f)=0, \\
& \left(-D_{t}^{2}+D_{x}^{2}+D_{y}^{2}+D_{z}^{2}+\tilde{a_{1}} \tilde{a_{2}}\right)(f \cdot f)=-g^{2}+\left(\tilde{a_{1}}+\tilde{a_{2}}\right) g f .
\end{aligned}
$$

Dividing the last equation on $f^{2}$ and using Eq. (37) we have quadratic algebraic equation on $U(\mathbf{x}, t)$

$$
U^{2}-\left(a_{1}+a_{2}\right) U+a_{1} a_{2}-2\left(\partial_{t}^{2}-\Delta\right) \ln f=0,
$$

where expression in the last parenthesis is the d'Alembert wave operator. Solution of this equation is given in terms of function $f(\mathbf{x}, t)$ only

$$
U(\mathbf{x}, t)=\frac{a_{1}+a_{2}}{2} \pm \sqrt{\frac{\left(a_{1}-a_{2}\right)^{2}}{4}+2\left(\partial_{t}^{2}-\Delta\right) \ln f(\mathbf{x}, t) .}
$$

After substituting the perturbative expansion (9) for $f$ and $g$, we obtain the zeroth, first and second order bilinear equations. The zeroth order equation has exactly the same (scalar) form with Eq. (13): $\left(g_{0}-\tilde{a}_{1}\right)\left(g_{0}-\tilde{a}_{2}\right)=0$. We choose solution as $g_{0}=\tilde{a}_{1}$. For the first order solution we assume $g_{1}=a e^{\eta_{1}}, f_{1}=b e^{\eta_{1}}$, where $\eta_{1}=\mathbf{k x}+\omega t+\delta_{0}$. Substituting into Eqs. (38a), we obtain the following dispersions, relating parameters $k$ and $\omega$

$$
\omega_{1}^{2}+\gamma \omega_{1}-\mathbf{k}_{1}^{2}-\tilde{a}_{1}\left(\tilde{a}_{2}-\tilde{a}_{1}\right)=0
$$

and

$$
\omega_{1}= \pm\left|\mathbf{k}_{1}\right| \text {. }
$$

We are not interested in the linear dispersion relation (42) since it leads to the constant solution. Thus, for the further calculations we use only relation (41). Eq. (41) has two roots:

$$
\omega_{1}^{(\mathrm{I}, \mathrm{II})}=-\frac{\gamma}{2} \pm \sqrt{\frac{\gamma^{2}}{4}+\mathbf{k}_{1}^{2}+\tilde{a_{1}}\left(\tilde{a_{2}}-\tilde{a_{1}}\right)} .
$$


Then, the ratio of parameters $a$ and $b$ is fixed as

$$
\frac{b}{a}=\frac{\left(\tilde{a_{2}}-\tilde{a_{1}}\right)}{2 \mathbf{k}_{1}^{2}+2 \omega_{1}^{2}+\tilde{a}_{1}\left(\tilde{a}_{2}-\tilde{a}_{1}\right)} .
$$

From the second order calculation, nonconstant solution exists only if this ratio is equal $a / b=\tilde{a}_{2}$. Substituting this value to Eq. (44) we find that wave vector $\mathbf{k}_{1}$ belongs to three dimensional sphere with fixed radius,

$$
\mathbf{k}_{1}^{2}=\frac{\left(\tilde{a}_{2}-\tilde{a}_{1}\right)^{2}}{2}\left[1+\frac{\left(\tilde{a}_{2}+\tilde{a}_{1}\right)^{2}}{2 \gamma^{2}}\right],
$$

while for the frequency we have only one value

$$
\omega_{1}=\frac{\tilde{a}_{2}^{2}-\tilde{a}_{1}^{2}}{2 \gamma}
$$

In the limit of strong dissipation, when $\gamma \gg 1$ the wave number (but not frequency) reduces to (36). In this limiting case the second time derivative term in Eq. (2) can be neglected and the equation reduces to the reaction-diffusion one.

Using explicit form for $f=1+b e^{\eta_{1}}$ and Eq. (40) we have solution in the form

$$
U=\frac{a_{1}+a_{2}}{2} \pm \frac{a_{1}-a_{2}}{2} \tanh \frac{\tilde{\eta_{1}}}{2}
$$

where $\tilde{\eta}_{1}=\mathbf{k}_{1} \mathbf{x}+\omega_{1} t+\delta_{0}+\ln b$. The velocity of our solution $\mathbf{v}=-\omega \mathbf{k} /|\mathbf{k}|^{2}$ is fixed by modulus

$$
|\mathbf{v}|=\frac{\frac{a_{1}+a_{2}-2 a_{3}}{\sqrt{2}}}{\sqrt{\left(\frac{a_{1}+a_{2}-2 a_{3}}{\sqrt{2}}\right)^{2}+\gamma^{2}}} .
$$

We note, that similarly to the pure diffusion case (36), when the root $a_{3}$ is equal to the mean value of other two roots $a_{3}=\left(a_{1}+a_{2}\right) / 2$, the velocity vanishes and the soliton becomes static. By cyclic permutation of indices $1,2,3$ in Eq. (47) we find other two solitons of our problem. But only one of them, which implement transition between stable vacuum states, could be stable.

\section{Nonlinear wave equation with transport term}

In this section, we consider nonlinear wave equation (3) with dissipation and nonlinear transport term. For simplicity we restrict here the analysis by the scalar equation with $n=1$. To apply the Hirota bilinearization we use the same as in previous section ansatz (37), and perturbation series expansion of $f$ and $g$. In terms of Hirota derivatives (5) we have the following bilinear system of equations

$$
\begin{aligned}
& \left(\alpha a_{3} D_{x}+\beta D_{t}^{2}+\gamma D_{t}-D_{x}^{2}\right)(g \cdot f)=0, \\
& \left(D_{x}^{2}-\beta D_{t}^{2}+\tilde{a_{1}} \tilde{a_{2}}\right)(f \cdot f)=-g^{2}+\left(\tilde{a_{1}}+\tilde{a_{2}}-\alpha D_{x}\right)(g \cdot f) .
\end{aligned}
$$

After applying the same procedure as before, we found the following dispersion relation:

$$
\beta \omega_{1}^{2}+\gamma \omega_{1}+\alpha a_{1} k_{1}-k_{1}^{2}+\left(a_{1}-a_{3}\right)\left(a_{1}-a_{2}\right)=0 .
$$

For the ratio of constants we obtain:

$$
\frac{b}{a}=\frac{-\alpha k_{1}-\left(a_{1}-a_{2}\right)}{\alpha k_{1}\left(a_{1}+a_{3}\right)+2 \gamma \omega_{1}+\left(a_{1}-a_{3}\right)\left(a_{1}-a_{2}\right)} .
$$

By equating this to $1 /\left(a_{2}-a_{3}\right)$ (to truncate the series), we get relation

$$
\omega_{1}=\frac{\tilde{a}_{2}^{2}-\tilde{a}_{1}^{2}}{2 \gamma}-\frac{\alpha\left(a_{1}+a_{2}\right)}{2 \gamma} k_{1} .
$$

Combining this equation with dispersion formula (50), we find quadratic equation fixing the wave number $k_{1}$ :

$$
A k_{1}^{2}+B k_{1}+C=0 \text {, }
$$


where

$$
\begin{aligned}
& A=\alpha^{2} \beta\left(a_{1}+a_{2}\right)^{2}-4 \gamma^{2}, \quad B=2 \alpha\left(a_{1}-a_{2}\right)\left[\gamma^{2}+\beta\left(a_{1}+a_{2}\right)\left(a_{1}+a_{2}-2 a_{3}\right)\right], \\
& C=\left(a_{1}-a_{2}\right)^{2}\left[2 \gamma^{2}+\beta\left(a_{1}+a_{2}-2 a_{3}\right)^{2}\right] .
\end{aligned}
$$

Substituting solution of this equation to (52), we find expression for $\omega_{1}$. The soliton solution is the shock wave

$$
U=a_{3}+\frac{\left(a_{1}-a_{3}\right)+\left(a_{2}-a_{3}\right) e^{\tilde{\eta}_{1}}}{1+e^{\tilde{\eta}_{1}}}
$$

implementing transition between $a_{1}$ and $a_{2}$ constant steady states. Since expressions are too long we are not presenting it in an explicit form. Instead of this in the next subsections, we reproduce $\omega_{1}$ and $k_{1}$, explicitly, for some special cases and calculate corresponding velocities of the soliton.

\subsection{Reaction-diffusion limit}

For $\alpha=0, \beta=0, \gamma=1$, Eq. (3) becomes of the nonlinear reaction-diffusion form

$$
\frac{\partial U}{\partial t}=\frac{\partial^{2} U}{\partial x^{2}}-\left(U-a_{1}\right)\left(U-a_{2}\right)\left(U-a_{3}\right)
$$

which corresponds also to the one dimensional $(d=1)$, scalar $(n=1)$ limit of the system (1). In this limiting case, from Eqs. (50), (51) and (53) for the wave scalar, the frequency and the velocity respectively, we have

$$
k=\mp \frac{a_{2}-a_{1}}{\sqrt{2}}, \quad \omega=\frac{\left(a_{2}-a_{3}\right)^{2}-\left(a_{1}-a_{3}\right)^{2}}{2}, \quad v= \pm \frac{a_{1}+a_{2}-2 a_{3}}{\sqrt{2}} .
$$

As easy to see, these formulas represent the one space dimensional reduction of Eqs. (36). When root $a_{3}$ is equal to the mean value of two other roots $a_{3}=\left(a_{1}+a_{2}\right) / 2$, the velocity vanishes and soliton is static. By cyclic permutation of indices 1,2,3 in Eqs. (54), we have another couple of kink solitons of our problem. If we order the real roots as $a_{1}<a_{3}<a_{2}$, then only two of the static homogeneous states $a_{1}$ and $a_{2}$ are stable and the corresponding kink (54) is stable.

Below we solve numerically the initial value problem for Eq. (55) with initial configuration in form of the step function determined by roots $a_{1}$ and $a_{2}$. We are using the explicit finite difference method and the Dirichlet boundary conditions. In a quite short time it leads to creation of one shock soliton. Depending on values of the roots $a_{3}$, the soliton is static or moving with fixed velocity.

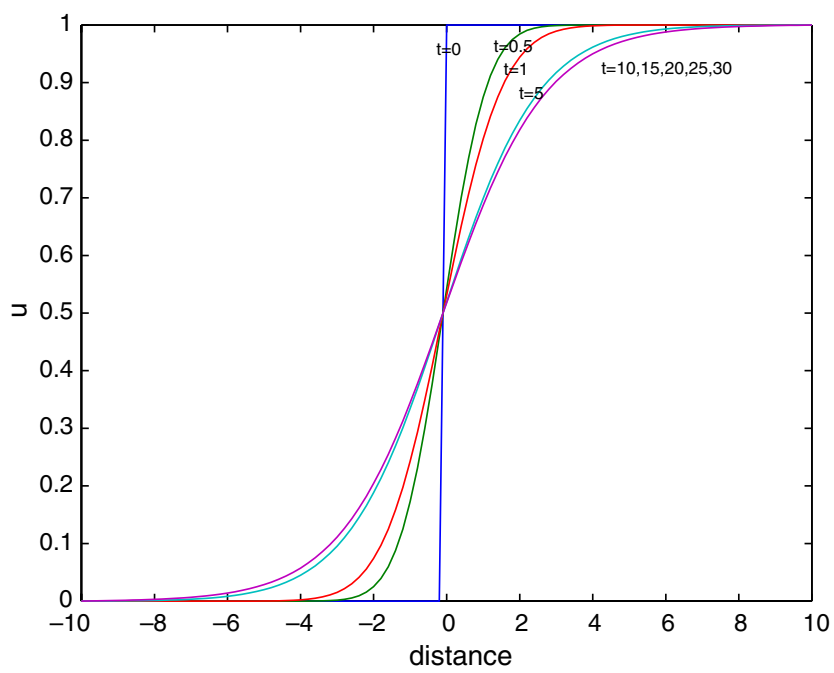

Fig. 1. Evolution of the travelling wave with $v=0$. The parameters $a_{1}=0, a_{2}=1, a_{3}=0.5$. 


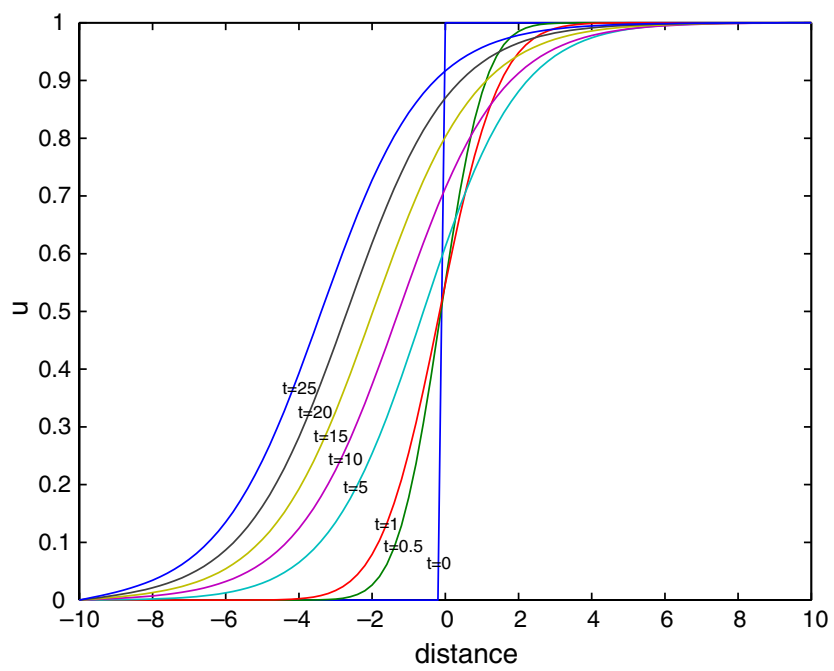

Fig. 2. Evolution of the travelling wave with $v=\frac{\sqrt{2}}{10}$. The parameters $a_{1}=0, a_{2}=1, a_{3}=0.4$.

In Fig. 1 the roots are considered as $a_{1}=0, a_{2}=1, a_{3}=0.5$. For those choice of parameters, the velocity calculated according Eq. (56) is vanishing $v=0$. As it can be seen in this figure, with such choice of parameters after $30 \mathrm{~s}$ there is no movement and generated shock soliton becomes static.

On the other hand, when we consider the roots as $a_{1}=0, a_{2}=1, a_{3}=0.4$, the velocity of the travelling wave in Eq. (56) is different from zero. In this case, the wave solution exhibited in Fig. 2 starting from time $25 \mathrm{~s}$ is moving with fixed shape and fixed velocity $v=\frac{\sqrt{2}}{10}$.

It is worth to note here that in contrast to the Burgers' equation, where velocity of shock soliton $v=\left(a_{1}+a_{2}\right) / 2$ is determined completely by parameters of the original step function $a_{1}, a_{2}$, in the present case the velocity includes the value $a_{3}$ (which is absent in the step function), so that for the same initial configuration we can have static or moving soliton, depending on that value.

\subsection{Burgers equation with cubic nonlinearity}

For $\beta=0$ and $\gamma=1$, Eq. (3) becomes of the Burgers form with cubic nonlinear term

$$
\frac{\partial U}{\partial t}+\alpha U \frac{\partial U}{\partial x}=\frac{\partial^{2} U}{\partial x^{2}}-\left(U-a_{1}\right)\left(U-a_{2}\right)\left(U-a_{3}\right) .
$$

In this case the dispersion relation (50) is reduced to

$$
\omega_{1}=k_{1}^{2}+\tilde{a_{1}}\left(\tilde{a_{2}}-\tilde{a_{1}}\right)-\left(\tilde{a_{1}}+a_{3}\right) \alpha k_{1},
$$

where $k_{1} \neq 0$, and the wave number is fixed as

$$
k_{1}=\frac{\left(\tilde{a}_{2}-\tilde{a}_{1}\right)\left(-\alpha \mp \epsilon \sqrt{\alpha^{2}+8}\right)}{4},
$$

where

$$
\epsilon= \begin{cases}1 & a_{2}>a_{1} \\ -1 & a_{2}<a_{1}\end{cases}
$$

The corresponding velocity of travelling wave is

$$
v=\frac{2\left(a_{1}+a_{2}-2 a_{3}\right)}{\beta}-\left(\frac{a_{2}+a_{1}}{2}\right) \alpha,
$$

where $\beta=-\alpha \pm \epsilon \sqrt{\alpha^{2}+8}$. This formula shows that for $\beta=\sqrt{2}$ amazingly, the velocity of shock is just sum of velocities for pure reaction-diffusion equation (56) and for the pure Burgers' equation. From the last equation one can see that even for the mean value condition $a_{3}=\left(a_{1}+a_{2}\right) / 2$ the solitary wave is not static. The static solitary wave arises for fixed 


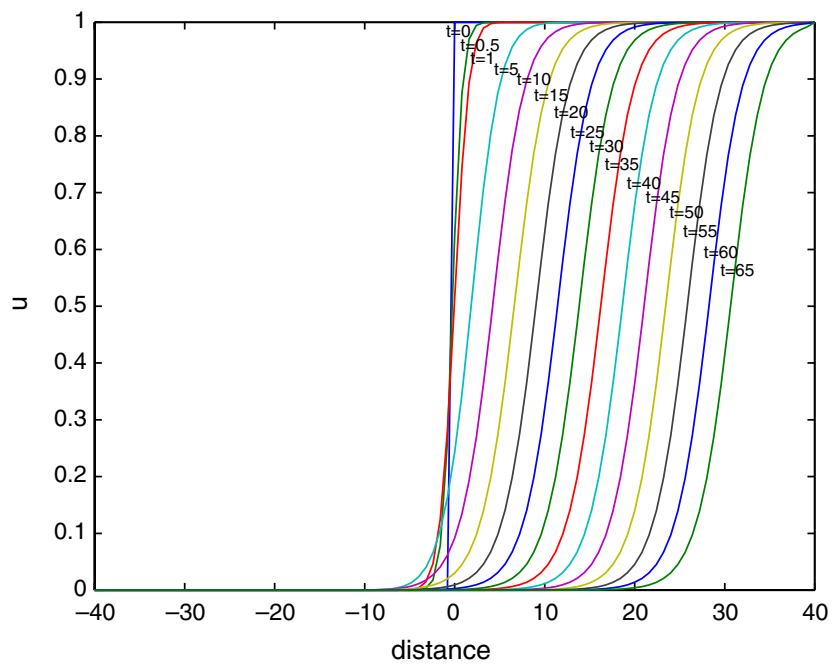

Fig. 3. Evolution of the travelling wave with $v \neq 0$. The parameters $a_{1}=0, a_{2}=1, a_{3}=0.5$

value of $\alpha$ in terms of $a_{1}, a_{2}, a_{3}$ in Eq. (60), such that $v=0$. Therefore by adding the nonlinear transport term to Eq. (55), we shifted the restriction on the roots of the polynomial to obtain moving solitary waves. By numerical solutions, similar to the previous static case, we consider one root of the third order polynomial as the mean of the other two, $a_{1}=0$, $a_{2}=1, a_{3}=0.5$. Fig. 3 shows that after time $25-30 \mathrm{~s}$ the shock soliton configuration is created with fixed shape and velocity, as expected from Eq. (60).

\section{Conclusion and discussion}

In the present paper, the Hirota method is applied to find exact analytic soliton solution of the nonlinear reactiondiffusion systems with multiple order parameter and with reaction part as the third order polynomial, in more then one space dimensions. To write equations in the bilinear form, we proposed to extract one of the background constant (unstable) solution (root) from Hirota's substitution. Truncation of the perturbation series in our second order calculations, restricts value of wave number and velocity of the travelling wave. In this sense, this truncation works similarly to the way Ablowitz and Zeppetella [6] obtained an exact travelling wave solution of Fisher's equation by finding the special wave speed for which the resulting ODE is of the Painleve type. Since bilinear form is written for equations in more than one space dimensions we expect to construct nonlinear wave configurations of amazing complexity, like circular or curved solitons, scroll-waves and vortex tubes. These questions are under our study now.

\section{Acknowledgment}

This work was supported partially by Izmir Institute of Technology grant 2002-IYTE-24 and 2002-IYTE-25.

\section{References}

[1] Murray JD. Mathematical biology, vols. I and II. Springer; 2003.

[2] Scott AS. Nonlinear science. Oxford University Press; 1999.

[3] Clarkson PA, Mansfield EL. In: Applications of analytic and geometric methods to nonlinear differential equations. Kluwer; 1993. p. 375-89.

[4] Cherniha RM. Rep Math Phys 1998;41:333-49.

[5] Khater AH, Malfliet W, Callebaut DK, Kamel ES. Chaos, Solitons \& Fractals 2002;14:513-22.

[6] Ablowitz MJ, Zeppetella A. Bull Math Biol 1979;41:835-40.

[7] Hirota R. Direct methods in soliton theory. In: Solitons. Springer-Verlag; 1980. 
[8] Miwa T, Jimbo M, Date E. Solitons: differential equations, symmetries and infinite dimensional algebras. Cambridge University Press; 2000.

[9] Aronson DG, Weinberger HF. In: Goldstein JA, editor. Partial differential equations and related topics. Lect Notes Math, vol. 446. Springer; 1975. p. 5-49

[10] Fitzhugh R. Biophys J 1961;1:445-66.

[11] Nagumo JS, Arimoto S, Yoshizawa S. Proc IRE 1962;50:2061-70.

[12] Schlögl F. Z Phys 1972;253:147-61;

Schlögl F, Berry RS. Phys Rev A 1980;21:2078-81.

[13] Dehrmann T. J Phys A: Math Gen 1982;15:L649-52.

[14] Newell AC, Whitehead JA. J Fluid Mech 1969;38:279-303.

[15] Kolmogoroff A, Petrovsky I, Piscounov N. Bull de l'Univ d'Etat a Moscou (Ser Int), A 1937;1:1-25.

[16] Kawahara T, Tanaka M. Phys Lett 1983;97A:311-4.

[17] Satsuma J. Exact solutions of Burgers' equation with reaction terms. In: Ablowitz M, Fuchssteiner B, Kruskal M, editors. Topics in soliton theory and exactly solvable nonlinear equations. World Scientific; 1986. 